OPEN ACCESS

Edited by:

Zikai He,

Harbin Institute of Technology, China

Reviewed by:

Xingchun Gao,

Xi'an Medical University, China

Qiang Cai,

Renmin Hospital of Wuhan University,

China

*Correspondence:

Longbo Zhang

longbo.zhang@yale.edu

${ }^{+}$These authors have contributed equally to this work

Specialty section:

This article was submitted to

Nanobiotechnology,

a section of the journal

Frontiers in Bioengineering and

Biotechnology

Received: 14 November 2021

Accepted: 10 December 2021

Published: 12 January 2022

Citation:

Zhang L, Getz SA and Bordey A (2022)

Dual in Utero Electroporation in Mice to

Manipulate Two Specific Neuronal

Populations in the Developing Cortex.

Front. Bioeng. Biotechnol. 9:814638.

doi: 10.3389/fbioe.2021.814638

\section{Dual in Utero Electroporation in Mice to Manipulate Two Specific Neuronal Populations in the Developing Cortex}

\author{
Longbo Zhang ${ }^{1,2 * t}$, Stephanie A. Getz ${ }^{2 \dagger}$ and Angelique Bordey ${ }^{2}$ \\ ${ }^{1}$ Departments of Neurosurgery, And National Clinical Research Center of Geriatric Disorders, Xiangya Hospital, Central South \\ University, Changsha, China, ${ }^{2}$ Departments of Neurosurgery, And Cellular and Molecular Physiology, School of Medicine, Yale \\ University, New Haven, CT, United States
}

Precise regulation of gene expression during development in cortical neurons is essential for the establishment and maintenance of neuronal connectivity and higher-order cognition. Dual in utero electroporation provides a precise and effective tool to label and manipulate gene expression in multiple neuronal populations within a circuit in a spatially and temporally regulated manner. In addition, this technique allows for morphophysiological investigations into neuronal development and connectivity following cell-specific gene manipulations. Here, we detail the dual in utero electroporation protocol.

Keywords: in utero electroporation, neuron, development, cortex, neuroonal connectivity

\section{INTRODUCTION}

In utero electroporation (IUE) is a powerful tool to target specific neuronal populations in the developing cortex with temporal and spatial resolution (Saito and Nakatsuji, 2001; Fukuchi-Shimogori and Grove, 2001). The protocol below describes a detailed protocol to perform dual IUE to target layers (L) $2 / 3$ and $4 / 5$ pyramidal neurons in the somatosensory cortex (SSC) with different fluorescent markers to study neuronal development, including dendrite growth and synaptic connectivity (Zhang et al., 2021) (Figure 1). To do this, DNA in a plasmid form is introduced into the lateral ventricle of embryos, and an electric pulse is applied to drive the DNA into the desired neural progenitor cell populations. IUE at embryonic day (E) 13.5 and 15.5 is targeted to neural progenitor cells that generate pyramidal neurons destined to reach L4/5 and L2/3, respectively (Molyneaux et al., 2007). Mechanistically, the current from the tweezertrode anode drives the negatively charged DNA toward the desired cortical region, while the electrical pulses generate pores to facilitate DNA delivery into the selected progenitor cell population. The DNA is then passed on to the daughter cells, i.e., neurons, from the progenitor cells upon cell division. These newborn neurons migrate to their target cortical layers and then differentiate into mature pyramidal neurons. Thus, a single IUE allows to manipulate a specific neuronal population within a discrete cortical location.

This protocol can be utilized to target different cortical regions, such as the medial prefrontal cortex (mPFC), as well as the hippocampus. In the DNA-encoding vector, different promoters can be used to restrict gene expression in neurons while bypassing expression in neural progenitor cells (e.g., doublecortin promoter; (Lin et al., 2016). This allows to selectively examine the impact of gene manipulations in neurons vs neural progenitor cells. Overexpression and short hairpin RNA-encoding vectors as well as CRISPR/Cas9 system can be used to examine the impact of gene overexpression, knockdown, or mutations on neuron development. Finally, the IUE procedure can be adapted to target unilateral or bilateral cortices. Taken together, dual IUE provides an excellent tool to target and manipulate one or multiple cell populations in a temporally- and spatially regulated manner to study neuronal development and ultimately connectivity, network activity, and animal behavior. Cortices can also be collected and plated for primary neuronal culture studies. 


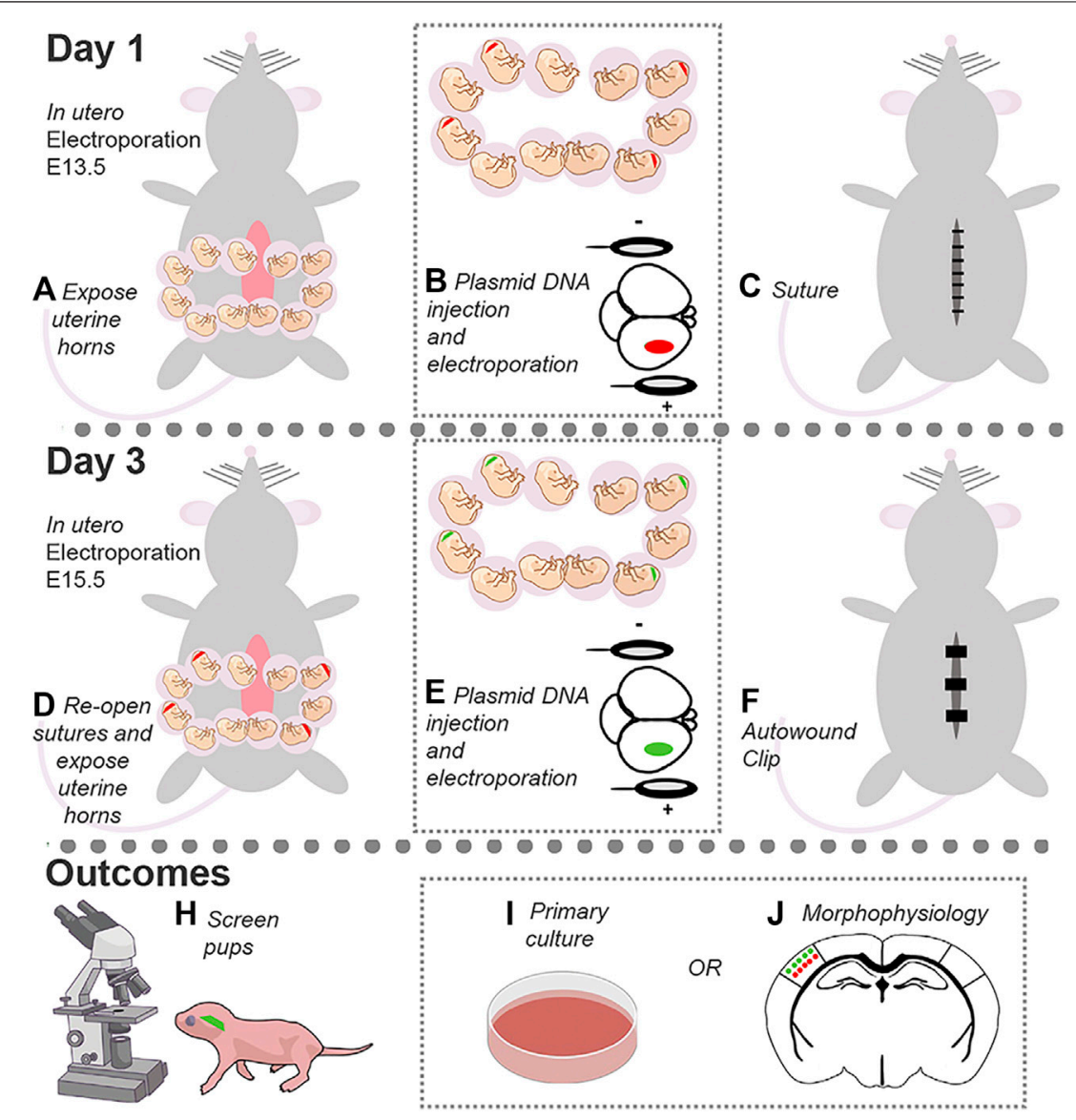

GRAPHICAL ABSTRACT |

\section{MATERIALS AND EQUIPMENT}

\section{Key Resources Table |}

Reagent or Resource

Source

Identifier

Chemicals, peptides, and recombinant proteins

Fast Green FCF

PBS, $1 \mathrm{x}, \mathrm{pH} 7.4$

Paraformaldehyde $32 \%$ Solution

Experimental models: Organisms/strains

CD-1 IGS Mice

Recombinant DNA

pCAG-GFP

pCAG-tdTomato

Sigma

Quality Biological

Electron Microscopy Sciences

Charles River Laboratories

Addgene

Addgene

Addgene

pCALNL-DsRed

pCAG-Cre

Surgical tools

Small Round-Tipped Forceps; 3 mm O.D., 2.2 mm I.D.

Large Round-Tipped Forceps; 6 mm O.D., 4.8 mm I.D.

Microdissecting Scissors; Straight; $23 \mathrm{~mm}$ Blade Length
Addgene

Hammacher Instrumente

Hammacher Instrumente

ROBOZ; SouthPointe Surgical

Supply
Ref F7252

Ref 114-058-101

Ref 15714-S

Ref 022

Addgene \# 11150; Matsuda et al. (2004) PNAS Addgene \# 83029; Pathania et al. (2012)

PLos One

Addgene \# 13769; Matsuda et al. (2007) PNAS Plasmid \# 13775; Matsuda et al. (2007) PNAS

Ref HSC_703-93

Ref HSC_703-96

Ref RS-5910SC 
Key Resources Table I (Continued)

\begin{tabular}{|c|c|c|}
\hline Reagent or Resource & Source & Identifier \\
\hline Webster Needle Holder; Serrated, Extra Delicate & WPI & Ref 14109 \\
\hline 5-0 UNIFY PGA Absorbable Braided, Coated Suture, Undyed; 18" & AD Surgical & Ref S-G518R13-U \\
\hline Autoclip $9 \mathrm{~mm}$ & Clay Adams Brand & Ref 300216 \\
\hline MikRon 9 mm AutoClip Applier & Clay Adams Brand & Ref NC9021392 \\
\hline \multicolumn{3}{|l|}{ Anesthesia system } \\
\hline $\begin{array}{l}\text { Vaporizor; EZ-7000 Classic System } \\
\text { Isoflurane; } 100 \mathrm{ml} \\
\text { IUE tools }\end{array}$ & $\begin{array}{l}\text { Vaporizor; EZ-7000 Classic System } \\
\text { Isoflurane; } 100 \mathrm{ml}\end{array}$ & $\begin{array}{l}\text { Vaporizor; EZ-7000 Classic System } \\
\text { Isoflurane; } 100 \mathrm{ml}\end{array}$ \\
\hline Electrode Puller & Narishige Japan & Model PP-830 \\
\hline $\begin{array}{l}\text { Borosilicate Glass with Filament; Fire Polished; O.D.: 1.5mm, I.D. } 1.10 \mathrm{~mm} \text {, } \\
10 \mathrm{~cm}\end{array}$ & Sutter Instrument & Ref BF150-110-10 \\
\hline Electro Square Porator; ECM 830 & BTX Harvard Apparatus & Ref W3 45-0052 \\
\hline Tweezertrodes Platinum Plated 3 mm & BTX Harvard Apparatus & Ref 45-0487 \\
\hline Tweezertrodes Platinum Plated 5 mm & BTX Harvard Apparatus & Ref 45-0489 \\
\hline Tweezertrodes Cable Adaptors & BTX Harvard Apparatus & Ref 45-0204 \\
\hline 15" Aspirator Tube Assembly & Drummond Scientific & Ref 2-000-000 \\
\hline \multicolumn{3}{|l|}{ Pre- and post-surgery treatment drugs } \\
\hline Rimadyl (carprofen); Injectable 50 mg/ml; 20 ml & Zoetis; Covetrus & Ref 024751 \\
\hline Buprenorphine $\mathrm{HCl}$ Injection: 0.3 mg/ml; C3 & Covetrus & Ref 059122 \\
\hline lidocaine $2.5 \%$ and prilocaine $2.5 \%$ Cream & Akorn; McKesson & Cat. No. 1331487 \\
\hline Puralube Vet Ointment & Dechra; Medvet & Cat. No. PH-PURALUBE-VET \\
\hline \multicolumn{3}{|l|}{ Screening and images collection } \\
\hline Fluorescence Microscope & Olympus & Ref SZX16 \\
\hline FV1000 & Olympus & \\
\hline Vibratome & Leica & Ref VT1000S \\
\hline \multicolumn{3}{|l|}{ Perfusion and fixation } \\
\hline Forceps for Perfusion & ROBOZ & RS-5136 \\
\hline Large scissors for Perfusion & WPI & Ref 19,520 \\
\hline Medium scissors for Perfusion & WPI & Ref 191210 \\
\hline Spring scissors for Perfusion & WPI & Ref 501235 \\
\hline Micro spatula for Perfusion & Sigma & Ref Z513377 \\
\hline \multicolumn{3}{|l|}{ Other } \\
\hline 0.9\% Sodium Chloride Injection; 10 ml & Hospira & Ref NDC 0409-4888-02 \\
\hline Sterile Empty Vial; $10 \mathrm{ml}$ & Hospira & Ref 5816-11 \\
\hline $30 \mathrm{G} \times 1 / 2(0.3 \mathrm{~mm} \times 13 \mathrm{~mm})$ PrecisionGlide Needle & Betcon, Dickinson and Company & Ref 305106 \\
\hline $1 \mathrm{ml}$ syringe; Tuberculin Slip Tip & Betcon, Dickinson and Company & Ref 309569 \\
\hline Polylined Sterile Field; Non-Fenestrated & Busse Hospital Disposables & Ref 696 \\
\hline Wahl Lithium-Ion Vacuum Trimmer Kit with Adjustable Vacuum Intake & Walmart & Cat. No. 9870 \\
\hline Non-Woven Sponges; Non-Sterile & McKesson & Ref 94442000 \\
\hline Prevantics & McKesson & Ref B108000 \\
\hline Alcohol Prep Pad & McKesson & Ref 58-204 \\
\hline 30 CC syringe; Luer Lock Tip; Sterile & McKesson & Ref $16-S 40 C$ \\
\hline Millex-GV Duapore PVDF Membrane; 0.22 um Filter unit & Millipore Sigma & Ref SLGVR33RS \\
\hline Acrodisc Syringe Filter; 0.2 um Supor Membrane Low Protein Binding & Pall Life Sciences & Ref 4602 \\
\hline Parafilm; 4 in $\times 125 \mathrm{ft}$ & Millipore Sigma & Ref P7793 \\
\hline Halogen Light Source & AmScope & Ref HL250 AY \\
\hline Snuggle Safe Heat Pad & Walmart & Cat. No. 596688379 \\
\hline
\end{tabular}

\section{METHODS}

\section{DNA Dilution Preparation}

Timing: 15 min.

1. Obtain DNA plasmids, filtered sterile PBS, and Fast Green $(0.25 \%)$ for electroporation.

a. Use a plasmid encoding a fluorescent protein under the CAG promoter (i.e., pCAG-tdTomato or pCAG-GFP; CAG is a cytomegalovirus [CMV] early enhancer fused to modified chicken actin promoter) pCAG-tdTomato or pCAG-GFP, to label neurons and detect efficient electroporation at birth in live mice.

Optional: use an inducible CAG promoter encoding DsRed following a floxed stop cassette (pCAG-LoxP-stop-LoxP-DsRed, called pCALNL-DsRed, addgene: \#13769) together with pCAG-GFP and low concentration of pCAG-Cre to label a subset of GFP-expressing neurons within the electroporated region (Zhang et al., 2021). 
Key Resources Table |

\begin{tabular}{lcl}
\hline DNA Dilution & & \\
\hline Reagent & $\begin{array}{c}\text { Final } \\
\text { concentration }\end{array}$ & \multicolumn{1}{c}{ Amount } \\
& & \\
\hline Plasmid DNA (pCAG-tdTomato) & $1.5 \mu \mathrm{g} / \mu \mathrm{L}$ & Depends on concentration \\
Plasmid DNA (pCAG-GFP) & $1.5 \mu \mathrm{g} / \mu \mathrm{L}$ & Depends on concentration \\
Fast green 0.25\% & $0.025 \%$ & $2.5 \mu \mathrm{L}$ \\
PBS 1x & $\mathrm{n} / \mathrm{a}$ & $\cup p$ to $10 \mu \mathrm{L}$ \\
Total & $\mathrm{n} / \mathrm{a}$ & $10 \mu \mathrm{L}$
\end{tabular}

Note: Make the day of surgeries and store at room temperature once made. One or more plasmids can be used per condition. Fast green concentration can be as low as $0.01 \%$. Optional: to label a subset of single neurons within the electroporated region, use a combination of plasmids pCALNL-DsRed and pCAG-Cre at 1000:1 ratio.

\begin{tabular}{lc}
\hline Key Resources Table I \\
\hline Electroporator Setup \\
\hline Electroporator parameters & Value \\
\hline Pulse Voltage & $36 \mathrm{mV}(\mathrm{E} 13.5) ; 39 \mathrm{mV}(\mathrm{E} 15.5)$ \\
Programmable Pulses & 5 \\
Pulse Width & $50 \mathrm{~ms}$ \\
Space Between Pulses & $950 \mathrm{~ms}$
\end{tabular}

\section{Key Resources Table |}

Anesthesia System Setup

\begin{tabular}{lcc}
\hline Anesthesia parameters & Isoflurane & LPM \\
\hline Induction & $4 \%$ & NA \\
Maintenance & $2 \%$ & NA \\
Primary Flowmeter & NA & 2.5 \\
Secondary Flowmeter & NA & 1.5
\end{tabular}

2. Combine reagents so that there is a total of $10 \mu \mathrm{L}$ plasmid DNA per mouse. DNA dilution can range from 1 to $4 \mu \mathrm{g} / \mu \mathrm{L}$. The table below uses $1.5 \mu \mathrm{g} / \mu \mathrm{L}$ as an example. Dilute fast green to a final concentration of $0.025 \%$. PBS will be used to bring the final volume to $10 \mu \mathrm{L}$.

3. Store stock DNA plasmids at $-20^{\circ} \mathrm{C}$ and working aliquots at $4^{\circ} \mathrm{C}$.

CRITICAL: Do not vortex DNA and avoid freeze-thawing. Flick the tube and spin down before use.

CRITICAL: Plasmid DNA concentration must be high enough, ideally, no lower than $5 \mu \mathrm{g} / \mu \mathrm{L}$, to allow for adequate expression in cells. Further, avoid thick plasmid DNA; if the DNA plasmid consistency is too viscous, then it will be challenging to use for electroporation.

\section{Electrode Preparation}

Timing: $15 \mathrm{~min}$.

4. Pull electrodes using a glass micropipette puller.

a. Have at least two electrodes per condition per animal when beginning surgeries.

b. If using a dual-stage glass micropipette puller (Narashige Model PP-830), then heat electrodes to $70.5{ }^{\circ} \mathrm{C}$ for these experiments. Use only the bottom electrode.
5. Place the micropipette tip inside the $6 \mathrm{~mm}$ O.D. large roundtipped forceps and gently break off the tip to create an angle of about $45^{\circ}$ (Figure 2). Break multiple sizes to test which tip diameter works best for each embryonic age.

CRITICAL: If the micropipette tip is too dull, then it will not penetrate the embryonic sac. If the tip is too thin, then it will be challenging to deposit plasmid into the brain. If there is clear damage to the cortex after electroporation, then the tip is likely too thick (Figure 2).

\section{Surgical Tool Preparation}

Timing: $1.5 \mathrm{~h}$.

6. Autoclave large and small round-tipped forceps, needle driver, and scissors. This can be completed the day of or the night before.

7. Place sterile $1 \mathrm{x}$ PBS into 30 - or 50-ml syringes fitted with a $0.22 \mathrm{~mm}$ filter for surgeries.

8. Sterilize fenestrated drapes and gauze.

\section{Primary IUE}

Timing: $0.5-1.0 \mathrm{~h}$.

1. Prewarm the heating pad in a microwave and $\mathrm{PBS}$ to $37^{\circ} \mathrm{C}$ in a water bath. Wipe down the surgical area with $70 \%$ ethanol.

2. Just before beginning surgery, administer $0.1 \mathrm{mg} / \mathrm{kg}$ body weight subcutaneous (s.c.) buprenorphine to timedpregnant mice.

3. Anesthetize a E13.5 timed-pregnant mouse by placing it in the isoflurane induction chamber.

a. Turn on oxygen, and open primary and secondary flowmeters. Set the secondary flowmeter to $~ 1.5$ LPM.

b. Turn on isoflurane to $4 \%$ and wait until the mouse is immobile, then decrease the level to $2 \%$.

4. After $\sim 3 \mathrm{~min}$, take the mouse out of the chamber and shave the mouse's belly with clippers while it is anesthetized.

Note: Suitable embryonic age can be differ depending on the regions. CRITICAL: If the mouse starts waking up after completion of the shaving procedure, place it back in the induction chamber and repeat Step 3.

5. Move the mouse onto the heating pad with its abdomen exposed and place its nose into the nose cone.

a. Put ophthalmic ointment on the eyes.

b. Place $2.5 \%$ lidocaine prilocaine cream on its abdomen and tape the limbs down.

6. Wipe down the abdomen using alternative swabs of prevantics and alcohol. Do this three times, starting with prevantics and ending with alcohol.

7. Cover the abdomen with a piece of fenestrated sterile drape and gauze.

a. Align the hole to be around the area of the incision.

b. Drench the gauze with the warm, sterile PBS.

8. Pinch the skin using the small ringed forceps and lift skin, make a skin incision of $\sim 2 \mathrm{~cm}$ long though the midline with scissors. 

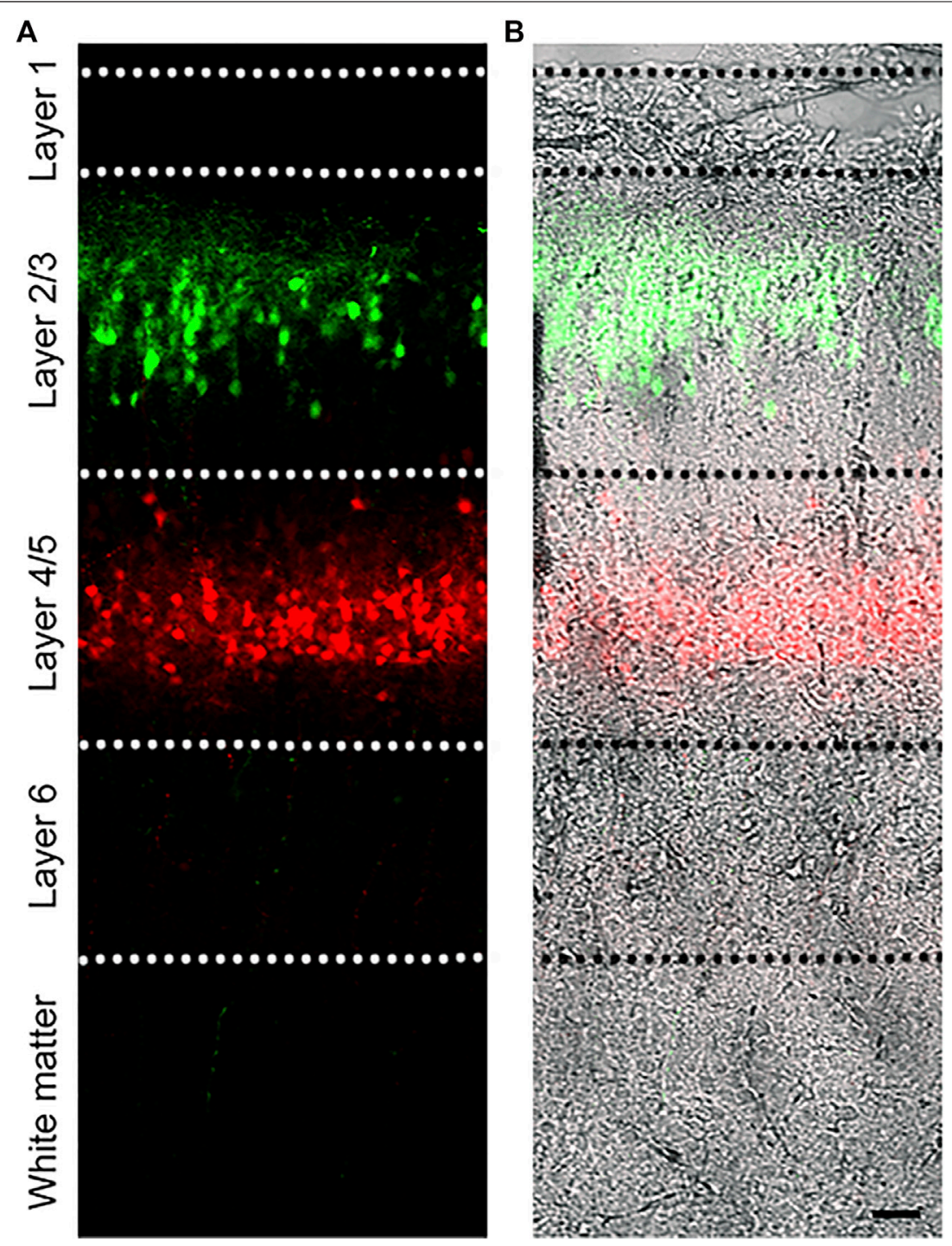

FIGURE 1 | Dual IUE image in the SSC. (A): Image of a postnatal day (P) 14 mouse coronal section containing GFP-expressing L2/3 cortical neurons and tdTomato-expressing L4/5 neurons following IUE at E15.5 and E13.5, respectively. (B): Fluorescent image overlaid with bright field. Bar: $50 \mu \mathrm{m}$.

9. Using the small ringed forceps, lift the muscle of abdominal wall and make an incision with scissors through the linea alba (white line) to avoid cutting of major blood vessels.

CRITICAL: Lifting the muscle before the incision of abdominal wall is important to avoid injuries of organs and embryos underneath. Begin with a small cut to allow the air in to push apart the abdominal wall and tissue underneath.

10. Gently move the uterine horns out through the incision using the large ring forceps and lay the uterine horns on the top of the gauze with the number of embryos counted and write the sequence of embryos in a notebook (Figure 3A). Regularly wet uterine horns with warm PBS.
CRITICAL: Carefully pull the uterine horns out of the abdominal cavity with the large ring forceps. Gently pull at the areas where embryos meet, rather than pulling directly on the embryos, to avoid damaging them. Alternatively, one can use fingers to gently move the embryos out while holding the peritoneum up and to the side with large ringed forceps.

11. Inject $1-2 \mu \mathrm{L}$ plasmid DNA solution into the lateral ventricle using a mouth-controlled micropipette, which consists of a $1 \mathrm{ml}$ syringe sans plunger, $15^{\prime \prime}$ aspirator tube assembly, $0.22 \mathrm{~mm}$ filter, and micropipette (Figure 3B).

Note: The lateral ventricle should be filled with fast green if the injection is successful (Figure 3B).

CRITICAL: To increase survival rate after the second IUE, inject embryos in an alternating pattern. 

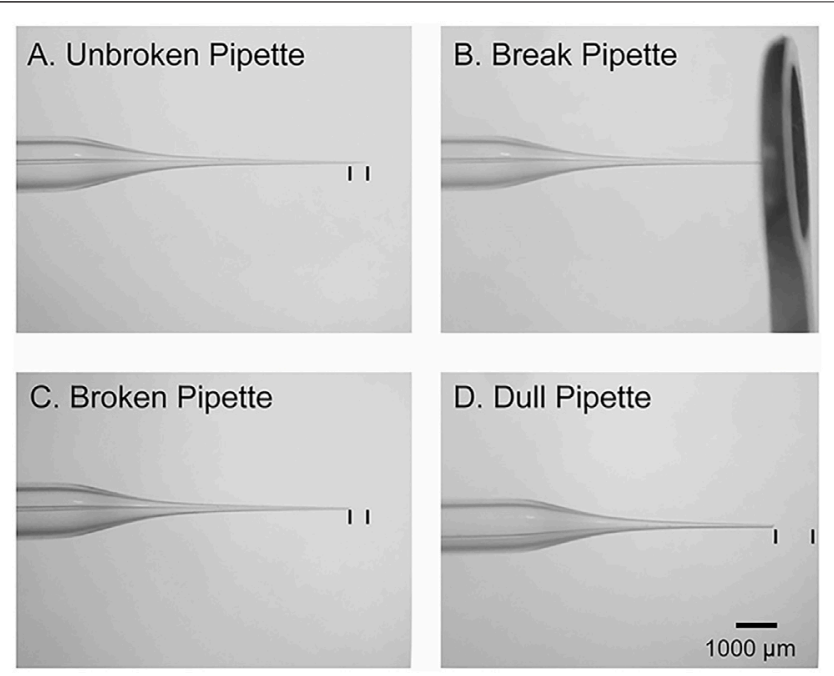

FIGURE 2 | Micropipette preparation. (A): Image of an intact glass micropipette that was generated by a vertical puller. The black lines denote the amount of tip that should be removed prior to insertion into the embryo. (B): Image of the large round forceps used to break the tip of micropipette.

(C): Image of an adequately broken micropipette with an 45 -degree-tip. The black lines denote the amount of tip that was removed. (D): An example of a dull pipette. The black lines denote the amount of tip that was removed.

CRITICAL: Avoid placing too much of the micropipette tip inside the embryonic sac. Doing so leads to holes, which can cause amniotic fluid to leak and increase the risk of abortion.

CRITICAL: Micropipette tips should not be reused for more than 10 embryos since the tip becomes blunt, which increases potential damage to embryos. Do not reuse electrodes across litters and conditions.

12. Place forceps-type electrodes (tweezertrode diameter: $2 \mathrm{~mm}$ ) parallel to DNA-injected embryos (position and direction of electrodes depend on the desired target areas) and deliver $36 \mathrm{mV}$ for $50 \mathrm{~ms}$-long electric pulses with a $950 \mathrm{~ms}$ pulse interval.

Note: The electrodes should be placed in PBS-containing conical tube to keep them wet.

CRITICAL: The tweezertrode anode should be placed to direct the DNA toward the progenitor cell population of interest. For the SSC, the tweezertrodes will be orthogonally oriented with the anode on the barrel cortex. The embryo should move slightly when electrical pulses are being applied.

CRITICAL: Gently push the embryo towards the embryonic sac to ensure more efficient electroporation delivery. The limbs of embryo should move after each electric pulse.

13. Repeat steps 11-12 for the desired embryos. Keep track of which embryos and hemispheres are electroporated.

CRITICAL: Regularly drop warm PBS onto the uterine horns before moving to the next electroporation.

14. Reposition the horns carefully using the large ring forceps back into the abdominal cavity. Fill cavity with warm PBS.

15. Suture the abdominal wall and skin with absorbable PDS suture line.

16. Cover the skin incision with triple antibiotic.

17. Administer $5 \mathrm{mg} / \mathrm{kg} / \mathrm{s.c}$. Rimadyl (carprofen).

18. Place the mouse in an empty cage on top of a heating pad until awake.

19. Upon waking, move the mouse back to its home cage. Put moistened food in the cage.

\section{Secondary IUE}

Timing: $0.5-1.0 \mathrm{~h}$.
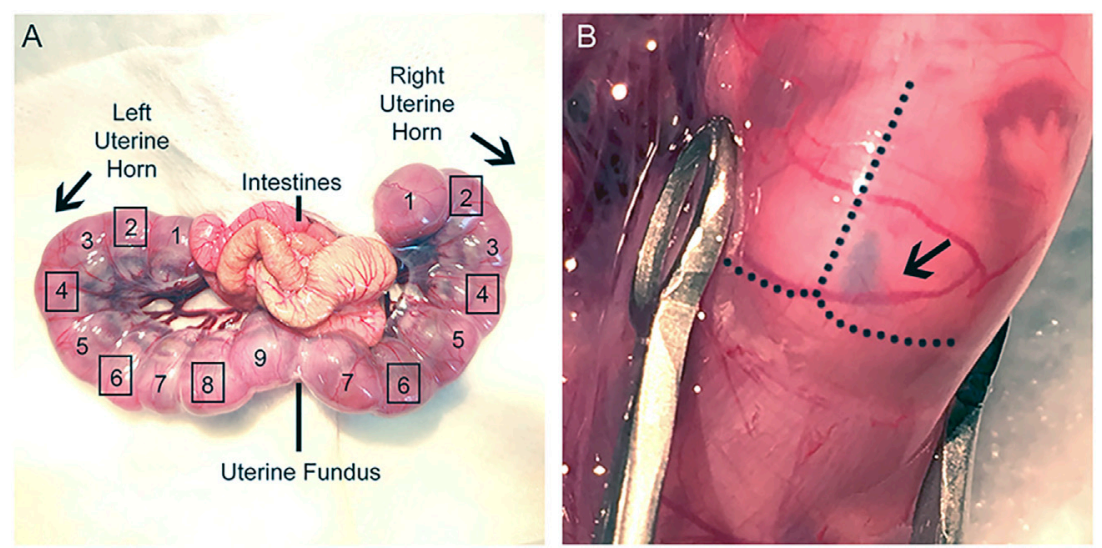

FIGURE 3 |Uterine horns exposure during IUE surgery. (A): Image of dissected uterine horns and labeling of desired IUE embryos. Uterine fundus is a useful marker to distinguish the two uterine horns and can be used as a reference to identify the electroporated embryos prior to the second IUE. Label embryos beginning at the uterine knots and continue sequentially to the uterine fundus for each uterine horn. Do not electroporate the embryos at the uterine knot or at the fundus (Left horn: 1, 9; Right horn: 1, 7). Squares surrounding the numbered embryos denote which embryos should receive IUE (Left horn: 2, 4, 6, 8; Right horn: 2, 4, 6). Electroporate embryos in an alternating fashion in the same hemisphere for both IUEs. (B): Image of lateral ventricle injection. Plasmid DNA was injected into E15 embryo, and the right lateral ventricle was filled with fast green. 

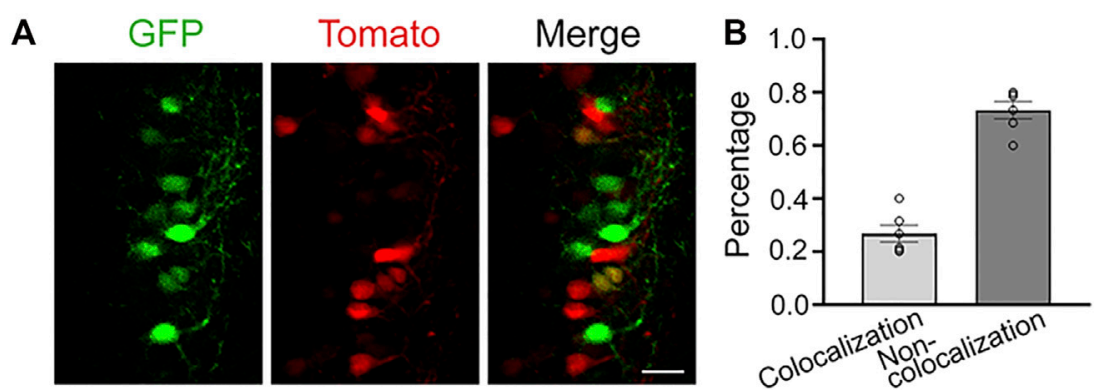

FIGURE 4 | Dual IUE 12-h apart leads to colocalization between the two electroporated cell populations. (A): Images of electroporated neurons in the SSC following a dual IUE 12-h apart (green: pCAG-GFP; red: pCAG-tdTomato). (B): Quantification of the GFP and RFP colocalization and non-colocalization. Bar: 25 um, 2 slices per mouse, $\mathrm{N}=3$ mice.

20. Monitor mouse at $24 \mathrm{~h}$ post-surgery and administer $5 \mathrm{mg} / \mathrm{kg} /$ s.c. Rimadyl (carprofen).

21. $48 \mathrm{~h}$ after the first IUE (E15.5), and $10 \mathrm{~min}$ prior to surgery, administer $0.1 \mathrm{mg} / \mathrm{kg} / \mathrm{s.c.}$ Buprenorphine.

CRITICAL: Performing the second IUE too soon (e.g., $12 \mathrm{~h}$ instead of $48 \mathrm{~h}$ ) following the first IUE results in a larger degree of co-localization between electroporated cell populations (Figure 4).

22. Anesthetize the mouse and repeat steps 1-7.

23. Using the small ringed forceps to pinch and lift skin, cut the suture line from the first surgery and gently open the original skin and abdominal wall incision.

Optional: Trim the skin and abdominal wall if needed.

24. Guide uterine horns out and lay them on the top of the gauze. Regularly wet uterine horns with warm PBS.

25. Find the electroporated embryos from last surgery and inject 1-2 $\mu \mathrm{L}$ plasmid DNA solution into the lateral ventricle using a micropipette. Make sure the lateral ventricle is filled with fast green.

26. Electroporate using forceps-type electrodes (Tweezertrodes diameter: $5 \mathrm{~mm}$; parameter setting: $39 \mathrm{mV}$ for $50 \mathrm{~ms}$-long electric pulses, $950 \mathrm{~ms}$ pulse interval). Make sure the embryos move with the electrical pulses.

27. After finishing the second IUE of the embryos, reposition the horns carefully using the large ring forceps into the abdominal cavity. Fill cavity with warm PBS.

28. Suture the abdominal wall with absorbable PDS suture line. Next, place one suture in the middle of the incision on the skin and staple the skin closed using autowound clips.

29. Repeat steps 16-19.

30. Post-surgical care: monitor mouse every day for $72-\mathrm{h}$ postsurgery and administer $5 \mathrm{mg} / \mathrm{kg} / \mathrm{s}$.c. Rimadyl (carprofen) daily.

\section{EXPECTED OUTCOMES}

Mice are typically born between E19 and E20. They can then be screened for fluorescence expression. IUE in CD1 mice are typically well-tolerated with $70-90 \%$ of electroporated pups being born. There could be some variability in the targeted region between mice and across litters. Practice can limit variability. In addition, including controls in the same litter is critical depending on the parameters (e.g., behavior) that are analyzed. Electroporated mice can be used for many purposes, including preparing fixed brain sections for imaging and morphological characterizations, preparing acute slices for electrophysiology, behavior analysis, and dissecting cells for in vitro experiments.

\section{DISCUSSION}

Dual IUE can target cortical neurons within the same cortical region but in different layers or target neurons in different cortical regions and layers. However, dual IUE may have a reduced efficacy compared to single IUE. One must be gentle with the embryos to maximize survival. When researchers are beginning this procedure, begin with a single IUE in the desired cortical location. Once it is established, then transition to dual IUE.

\section{Troubleshooting \\ Problem 1}

Cannot penetrate the embryonic sac with the micropipette.

\section{Potential Solution}

The micropipette tip is too dull or wide. Change to a sharper tip and repeat. Proceeding with a micropipette tip that is too dull or wide could damage the embryo (Figure 2).

\section{Problem 2}

Cannot deposit the plasmid into the lateral ventricle.

\section{Potential Solution}

There could be multiple causes for this (Saito and Nakatsuji, 2001): The tip has bypassed or passed through the lateral ventricle and is somewhere else in the brain. Pull back on the tip while continuing to gently blow out the plasmid (FukuchiShimogori and Grove, 2001). The micropipette tip is too thin to inject DNA solution into lateral ventricle. Change 
micropipette to a slightly larger tip diameter (Zhang et al., 2021). There is too much pressure on the pup. Loosen the grip on the pup and try again.

\section{Problem 3}

The embryonic sac is leaking amniotic fluid.

\section{Potential Solution}

The micropipette tip may be too dull or wide. Adjust micropipette sharpness or thickness accordingly (Fukuchi-Shimogori and Grove, 2001). The micropipette was placed too deep into the embryonic sac during plasmid injection. Ensure the micropipette is properly inserted in the lateral ventricle.

\section{Problem 4}

Popping of the embryonic sac during handling.

\section{Potential Solution}

If this happened, the embryos will not likely survive. Handle the embryos more gently.

\section{Problem 5}

The dam is bleeding.

\section{Potential Solution}

Although this procedure should not result in excessive bleeding, there could be blood between the skin and peritoneum. As long as it is only a small amount of blood, this will resolve as the surgery is completed. If an organ was ruptured, then there would be more blood within the peritoneal cavity. If so, euthanize the animal.

\section{Problem 6}

Few or no electroporated pups are born.

\section{Potential Solution}

The experimenter was likely not gentle enough with the embryos during surgery. The experimenter should put less pressure on the embryos, deposit the DNA plasmids with less force, and consider altering micropipette sizes. In addition, pups at the top of the uterine horn (near the knots) and at the junction between the two uterine horns should not be electroporated otherwise this increases the chances of spontaneous abortions.

\section{Problem 7}

Electroporated pups are born, but the fluorescent protein is expressed in subcortical or incorrect cortical locations.

\section{Potential Solution}

If the fluorescent reporter is in subcortical locations, then the micropipette was too deep. Ensure the plasmid DNA is properly injected into the lateral ventricle. If it is expressed in incorrect cortical locations, then adjust tweezertrode orientation to obtain the correct location.

\section{Problem 8}

Electroporated pups are born, but there is noticeable damage to the cortex or hydrocephalus.

\section{Potential Solution}

If there is damage to the cortex, then the micropipette was too dull. Hydrocephalus can happen with IUE, but it is uncommon. This could occur for several reasons: 1) too much pressure when depositing the plasmid into the lateral ventricle, 2) from the micropipette injection causing bleeding inside the lateral ventricle, or 3) inflammation. Ensure sterilizing the surgical and IUE tools before start, and deposit plasmid DNA gently.

\section{Problem 9}

Only some of the pups have dual IUE, while others have a single IUE.

\section{Potential Solution}

This is likely because one of the IUEs was unsuccessful, or the second IUE was performed out of sequence and did not target the electroporated embryos. Some embryos could die between the first and second IUE. A dying embryo has an opaque whiteyellowish color prior to being fully resorbed. Be mindful to count all the embryos carefully during the first and second IUEs, and note which embryos are no longer healthy. Skip unhealthy or dying embryos, even if they were part of the first IUE. When skipping those dying embryos, make sure to remain in sequence with the previous IUE pattern so that all healthy embryos that received the first IUE (E13.3) also receive the second IUE (E15.5).

\section{DATA AVAILABILITY STATEMENT}

The original contributions presented in the study are included in the article/Supplementary Material, further inquiries can be directed to the corresponding author.

\section{ETHICS STATEMENT}

The animal study was reviewed and approved by Yale University Institutional Animal Care and Use Committee.

\section{AUTHOR CONTRIBUTIONS}

All authors listed have made a substantial, direct, and intellectual contribution to the work and approved it for publication

\section{FUNDING}

The work was funded by a National Natural Science Foundation of China 82171171 (LZ), Tuberous sclerosis complex Alliance research grant $(\mathrm{AB})$. 


\section{REFERENCES}

Fukuchi-Shimogori, T., and Grove, E. A. (2001). Neocortex Patterning by the Secreted Signaling Molecule FGF8. Science 294 (5544), 1071-1074. doi:10.1126/science.1064252

Lin, T. V., Hsieh, L., Kimura, T., Malone, T. J., and Bordey, A. (2016). Normalizing Translation through 4E-BP Prevents mTOR-Driven Cortical Mislamination and Ameliorates Aberrant Neuron Integration. Proc. Natl. Acad. Sci. USA 113 (40), 11330-11335. doi:10.1073/pnas.1605740113

Molyneaux, B. J., Arlotta, P., Menezes, J. R. L., and Macklis, J. D. (2007). Neuronal Subtype Specification in the Cerebral Cortex. Nat. Rev. Neurosci. 8 (6), 427-437. doi:10.1038/nrn2151

Saito, T., and Nakatsuji, N. (2001). Efficient Gene Transfer into the Embryonic Mouse Brain Using In Vivo Electroporation. Developmental Biol. 240 (1), 237-246. doi:10.1006/dbio.2001.0439

Zhang, L., Zhang, X., Hsieh, L. S., Lin, T. V., and Bordey, A. (2021). Rab27a-Dependent Paracrine Communication Controls Dendritic Spine Formation and Sensory Responses in the Barrel Cortex. Cells 10 (3), 622. doi:10.3390/cells10030622
Conflict of Interest: The authors declare that the research was conducted in the absence of any commercial or financial relationships that could be construed as a potential conflict of interest.

Publisher's Note: All claims expressed in this article are solely those of the authors and do not necessarily represent those of their affiliated organizations, or those of the publisher, the editors and the reviewers. Any product that may be evaluated in this article, or claim that may be made by its manufacturer, is not guaranteed or endorsed by the publisher.

Copyright $\odot 2022$ Zhang, Getz and Bordey. This is an open-access article distributed under the terms of the Creative Commons Attribution License (CC BY). The use, distribution or reproduction in other forums is permitted, provided the original author(s) and the copyright owner(s) are credited and that the original publication in this journal is cited, in accordance with accepted academic practice. No use, distribution or reproduction is permitted which does not comply with these terms. 